\title{
Asymptotic normality of non-parametric estimator for the FGT poverty index with when the parameter is strictly between 0 and 1
}

\author{
Youssou Ciss and Aboubakary Diakhaby \\ LERSTAD, UFR de Sciences Appliquées et de Technologie, B.P 234 Université Gaston Berger de \\ Saint-Louis, Sénégal
}

Received August 28, 2016; Accepted September 5, 2016

Copyright (C) 2016, Afrika Statistika and Statistics and Probability African Society (SPAS). All rights reserved

\begin{abstract}
In this paper, we study the kernel estimator of Foster, Greer and Thorbecke class of measures when the poverty aversion parameter is strictly between zero and one, as a generalization of the work of Dia (2009). We solved an open problem arising in mentioned paper. The asymptotic normality of the estimator is established. As an illustration, we determine the confidence intervals for different regions of Senegal. The study of this application demonstrated that our methodology is not only more efficient than the empirical estimator, but it also provides better confidence intervals for the poverty index.

Résumé. Dans ce papier, nous étudions l'estimateur à noyau de la classe de mesures de Foster, Greer et Thorbecke pour généraliser les résultats de Dia (2009) pour de petites valeurs du paramètre d'aversion, c'est à dire lorsque ce dernier est compris entre 0 et 1 strictement. Ce cas restait un problème ouvert dans le travail de Dia. Nous avons établi la normalité asymptotique de l'estimateur à noyau. A titre d'illustration, nous déterminerons les intervalles de confiance pour les différentes régions du Sénégal. Nous montrons par cette application que l'estimateur proposé est non seulement plus efficace que l'estimateur empirique mais, il produit aussi des intervalles de confiance plus petits de contenant l'indice de pauvreté.
\end{abstract}

Key words: Poverty line; Poverty aversion; Moving kernel; Foster; Greer and Thorbecke; Uniform convergence; Asymptotic normality.

AMS 2010 Mathematics Subject Classification : Primary 60F05; Secondary 62G05.

${ }^{*}$ Corresponding author Youssou Ciss: ciss.youssouf@gmail.com Aboubakary Diakhaby :diakhaby@ugb.edu.sn, diakhaby81@yahoo.fr 
Y. Ciss and A. Diakhaby, Afrika Statistika, Vol. 11(2), 2016, pages 965-980. Asymptotic normality of non-parametric estimator for the FGT poverty index with when the parameter is strictly between 0 and 1

\section{Introduction and definition of the estimator}

The results presented below are mainly inspired by the work of G. Dia in which he established his results for $\alpha=0$ and $\alpha \geq 1$ Dia (2009). In our case, we give the same results but for $\alpha$ strictly between 0 and 1 .

Let $F(x)$ be the cumulative distribution function (cdf) of the income variable $X$ from a population with continuous density (pdf) $f(x)$ at a point $x$ on a given probability space $(\Omega, \mathcal{A}, \mathbb{P})$. The FGT Foster et al. (1984) class of poverty measures indexed by $\alpha \geq 0$ is defined by

$$
P(z, \alpha)=\left\{\begin{array}{rr}
\int_{0}^{z}\left(\frac{z-x}{z}\right)_{0}^{\alpha} f(x) d x & \text { if } z>0 \\
\text { otherwise }
\end{array}\right.
$$

where $z$ is the poverty line.

Lo (2003) in all his first reseach studies this measure in the following form

$$
P_{\alpha, H}=\frac{1}{H} \sum_{i \in Q(x)}\left(\frac{z-x_{i}}{z}\right)^{\alpha}
$$

for $H=n$ and $H=q$. He showed using the sampling tools that the estimators were respectively unbiased and asymptotically unbiased.

Now let us consider, for an integer $n \geq 1$, a random sample $\left(X_{1}, \ldots, X_{n}\right)$ from $X$, defined on the probability space defined above. The empirical estimator of (1) is given by

$$
\widehat{P}_{n}(z, \alpha)=\frac{1}{n} \sum_{i=1}^{n}\left(1-\frac{X_{i}}{z}\right)_{+}^{\alpha} \quad \text { where } \quad x_{+}=\max (0, x) \quad \text { Seidl (1988). }
$$

This empirical estimator is unbiased consistent and has a limit normal law with zero mean and variance equal to $n^{-1}\left(P(z, 2 \alpha)-(P(z, \alpha))^{2}\right)$. Therefore, the statistic

$$
T=\sqrt{n} \frac{\widehat{P}_{n}(z, \alpha)-P(z, \alpha)}{\sqrt{\left(\widehat{P}_{n}(z, 2 \alpha)-\left(\widehat{P}_{n}(z, \alpha)\right)^{2}\right)}}
$$

can be used to built confidence interval for the poverty measure (see Dia, 2009). So, asymptotic normal estimator with smaller variance will be in general preferable to $\widehat{P}_{n}(z, \alpha)$.

Lo et al. (2009) used the empirical process theory and the extreme value theory to study this estimator. This family has been showed to be both a Glivenko-Cantilli and a Donsker one, as a particular case of the family

$$
\widehat{P}_{n}(z, \alpha, g)=\frac{1}{n} \sum_{i=1}^{Q} g\left(1-\frac{X_{i, n}}{z}\right)^{\alpha}
$$

where $g$ lies in a suitable family of functions $\mathcal{G}$, and $X_{1, n} \leq \ldots \leq X_{n, n}$ denote the order statistics associated with $X_{1}, \ldots, X_{n}$. Seck (2011) and Seck and, Lo (2009) use some nonweighted poverty measures, viewed as stochastic processes and indexed by real numbers or monotone functions, to follow up the poverty evolution between two periods. But earlier 
Y. Ciss and A. Diakhaby, Afrika Statistika, Vol. 11(2), 2016, pages 965-980. Asymptotic normality of non-parametric estimator for the FGT poverty index with when the parameter is strictly between 0 and 1

in 2008, Dia (2008) introduced the kernel based estimation of (1) by supposing that the distribution function has a probabily density function $f$. Then replacing $f$ by its ParzenRosenblatt kernel estimator, he got the kernel FGT estimator, that he was able to describe in terms of uniform almost sure convergence and uniform squared mean convergence for $\alpha=0$ and $\alpha \geq 1$. The case $\alpha \in] 0,1[$ remained an open problem.

The classical estimator of the density $f$ (Parzen-Rosenblatt) given by

$$
\hat{f}(x)=\frac{1}{n} \sum_{i=1}^{n} \frac{1}{h} K\left(\frac{x-X_{i}}{h}\right) .
$$

In a previous paper Ciss et al. (2015), Ciss et al. have proposed a new method of kernel estimate based on Riemann sum, namely the following estimator

$$
P_{n}(z, \alpha)=\frac{1}{n} \sum_{k=1}^{n} \sum_{i=1}^{\left[\frac{z}{h}\right]}\left(1-\frac{i h}{z}\right)^{\alpha} K\left(\frac{X_{k}-i h}{h}\right)
$$

where $[\dot{\bar{h}}]$ stands for the largest integer smaller than or equal to $\dot{\bar{h}}$ and for $\alpha \in] 0,1[$.

Our contribution was to prove that this kernel estimator introduced by Dia can be used for each value of index $\alpha \in[0, \infty[$, which is important since the FGT measures concerns tests for poverty ordering or, equivalently, stochastic dominance and also optimal policy design (or program) for reducing poverty where the case $\alpha<1$ is specific and useful as pointed out in Foster et al. (2010).

In (1) $h=h(n)$ is a positive nonrandom sequences of real numbers tending to zero as $n$ tends to infinity, and finally $K$, a Riemann integrable kernel which satisfies the following hypotheses:

$$
\left(\mathbf{H}_{1}\right) \sup _{-\infty<x<+\infty}|K(x)|<+\infty,\left(\mathbf{H}_{\mathbf{2}}\right) \int_{-\infty}^{+\infty} K(x) d x=1,\left(\mathbf{H}_{\mathbf{3}}\right) \lim _{x \rightarrow \pm \infty}|x||K(x)|=0 .
$$

The rest of the paper is organized as follows. In section 2, we will state full details of the results. In section 3, as an illustration, we will determine the confidence intervals for different regions of Senegal and revelant comments as well as a comparaison with results from the empirical approch that was used until now. The complete proofs are then given in section 4 .

\section{Asymptotic Normality}

We will need a number of hypotheses and conditions for our theorems.

Now additional hypotheses on $\mathbb{K}$ or $K$ are the following:

$\left(\mathbf{H}_{4}\right) K$ is of bounded variation function $V_{-\infty}^{u} K$ on $\mathbb{R}$ and we denote by $V(\mathbb{R})$ its total variation.

$\left(\mathbf{H}_{\mathbf{5}}\right) \int_{\mathbb{R}}|u||K(u)|<+\infty$.

$\left(\mathbf{H}_{\mathbf{6}}\right)$ There exists a nonincreasing function $\lambda$ such as $\lambda\left(\frac{u}{h}\right)=O(h)$ on any bounded interval and for two real numbers $x$ and $y$

$$
|K(x)-K(y)| \leq \lambda|x-y| \quad \text { and } \quad \lambda(u) \longrightarrow 0, u \rightarrow 0, u \geq 0,
$$


Y. Ciss and A. Diakhaby, Afrika Statistika, Vol. 11(2), 2016, pages 965-980. Asymptotic normality of non-parametric estimator for the FGT poverty index with when the parameter is strictly between 0 and 1

$\left(\mathbf{H}_{\mathbf{7}}\right) \frac{|x|}{|h|^{1+\varepsilon}}\left|K\left(\frac{x}{h}\right)\right| \rightarrow 0, \quad 0<\varepsilon<\frac{1}{2}$, as $\frac{|x|}{h} \rightarrow+\infty$.

Next, these conditions depend of the $p d f f(x)$ :

$\mathbf{C}_{\mathbf{1}}: f(x)$ is uniformly continuous.

$\mathbf{C}_{\mathbf{2}}: f(x)$ admits almost everywhere a derivative $f^{\prime}(x) \in L_{1}(\mathbb{R})$.

$\mathbf{C}_{3}: f(x)$ satisfies a $C$-Lipschitz condition of order $\gamma, \quad 0<\gamma \leq 1$.

Under hypothesis $\mathbf{C}_{\mathbf{1}}$ or $\mathbf{C}_{\mathbf{2}}$, the convergence of the above estimator was established in Ciss et al. (2015).

Finally, We consider a family of kernels $K_{\nu}, \quad \nu \in \Gamma \subset \mathbb{R}_{+}^{*}, \quad \mathbb{R}_{+}^{*}$ being the set of strictly positive real numbers, about which we made respectively the same hypotheses $\mathbf{H}_{\mathbf{1}}-\mathbf{H}_{\mathbf{7}}$. We denote by $P_{n}^{\nu}$ the estimator of $P(z, \alpha)$ when we replace $K$ by $K_{\nu}$ in $P_{n}$. We suppose $\int_{\mathbb{R}} K_{\nu}^{2}(y) d y<1$ for all $\quad \nu \in \Gamma$ and $\sup _{\nu \in \Gamma} \int_{\mathbb{R}} K_{\nu}^{2}(y) d y=1$. Let $N(0,1)$ be the standard normal distribution function. Our main resultats are the following:

Theorem 1. Assume the hypotheses $\mathbf{C}_{\mathbf{3}}, \mathbf{H}_{\mathbf{6}}, \mathbf{H}_{\mathbf{7}}$ hold and $\int_{\mathbb{R}}\left|y^{\gamma} K_{\nu}^{2}(y)\right| d y<+\infty$ for all $\nu \in \Gamma$. If $n h^{2 \gamma} \rightarrow 0$ when $n \rightarrow+\infty$, then

$$
\frac{P_{n}^{\nu}(z, \alpha)-P(z, \alpha)}{\sqrt{\operatorname{Var}\left(P_{n}^{\nu}(z, \alpha)\right)}} \rightarrow N(0,1)
$$

in distribution as $n \rightarrow+\infty$, provided

$$
\left(\int_{\mathbb{R}} K_{\nu}^{2}(y) d y\right) P(z, 2 \alpha)-(P(z, \alpha))^{2}>0 .
$$

Theorem 2. Assume the hypotheses $\mathbf{C}_{\mathbf{2}}, \mathbf{H}_{\mathbf{5}}, \mathbf{H}_{\mathbf{7}}$ hold. If $n h^{2 \gamma} \rightarrow 0$ when $n \rightarrow+\infty$, then

$$
\frac{P_{n}^{\nu}(z, \alpha)-P(z, \alpha)}{\sqrt{\operatorname{Var}\left(P_{n}^{\nu}(z, \alpha)\right)}} \rightarrow N(0,1)
$$

in distribution as $n \rightarrow+\infty$, provided

$$
\left(\int_{\mathbb{R}} K_{\nu}^{2}(y) d y\right) P(z, 2 \alpha)-(P(z, \alpha))^{2}>0 .
$$

We establish these two theorems by proving the two following lemmas which are respectively a generalisation in three dimensions of the function $K$ in Lemma 2.9 and in Theorem 2.10 of Ciss et al. (2015).

Lemma 1. Let $0 \leq \theta_{i} \leq 1 ; \quad i=1,2,3$. Then for all $x, y$, $t$ pairwise different we have

$$
\begin{array}{r}
\operatorname{sim}_{n \rightarrow+\infty} \sup _{\left(\theta_{1}, \theta_{2}, \theta_{3}\right) \in[0,1] \times[0,1] \times[0,1]}\left(h^{-3} \int_{-\infty}^{+\infty} \mid K\left(\frac{u-x+\theta_{1} h}{h}\right) K\left(\frac{u-y+\theta_{2} h}{h}\right)\right. \\
\left.\times K\left(\frac{u-t+\theta_{3} h}{h}\right) \mid f(u) d u\right)=0 .
\end{array}
$$

Journal home page: www.jafristat.net 
Y. Ciss and A. Diakhaby, Afrika Statistika, Vol. 11(2), 2016, pages 965-980. Asymptotic normality of non-parametric estimator for the FGT poverty index with when the parameter is strictly between 0 and 1

Lemma 2. Assume hypothese $\mathbf{C}_{\mathbf{1}}$ or $\mathbf{C}_{\mathbf{2}}$ holds. Then under hypotheses $\mathbf{H}_{\mathbf{6}}$ and $\mathbf{H}_{\mathbf{7}}$ we have for all $b>0$,

$$
\begin{array}{r}
\lim _{n \rightarrow+\infty} \sup _{z \in[0, b]} \sum_{0 \leq i \neq j \neq l \neq i \leq\left[\frac{z}{h}\right]}\left(1-\frac{i h}{z}\right)^{\alpha}\left(1-\frac{j h}{z}\right)^{\alpha}\left(1-\frac{l h}{z}\right)^{\alpha} \int_{\mathbb{R}} K\left(\frac{u-i h}{h}\right) K\left(\frac{u-j h}{h}\right) \\
\times K\left(\frac{u-l h}{h}\right) f(u) d u=0 .
\end{array}
$$

Remark 1. To construct a confidence interval we proceed as follows:

For $0<\beta<1$, let $q_{1-\frac{\beta}{2}}$ be the $\beta$-quantile of the standardized normal distribution. Since $\operatorname{Var}\left(P_{n}^{\nu}(z, \alpha)\right) \geq 0$ for all $z$ and by the Theorems 2.7 and 2.8 in Ciss et al. (2015)

$$
\lim _{n \rightarrow+\infty} n \operatorname{Var}\left(P_{n}^{\nu}(z, \alpha)\right)=\left(\int_{\mathbb{R}} K_{\nu}^{2}(y) d y\right) P(z, 2 \alpha)-(P(z, \alpha))^{2},
$$

we have $\lim _{n \rightarrow+\infty} n \operatorname{Var}\left(P_{n}^{\nu}(z, \alpha)\right)=0$ for all $z$ such that $\left(\int_{\mathbb{R}} K_{\nu}^{2}(y) d y\right) P(z, 2 \alpha)-$ $(P(z, \alpha))^{2} \leq 0$. It follows that the asymptotic efficiency $e^{K_{\nu}}(z, \alpha)$ verifies

$$
0 \leq e^{K_{\nu}}(z, \alpha)=\lim _{n \rightarrow+\infty} \frac{n \operatorname{Var}\left(P_{n}^{\nu}(z, \alpha)\right)}{n \operatorname{Var}\left(\widehat{P}_{n}(z, \alpha)\right)} \leq \int_{\mathbb{R}} K_{\nu}^{2}(y) d y<1
$$

for conventional kernels in Parzen (1962) p.1068.

We define $100(1-\beta) \%$ the confidence interval $C I_{\nu}$ for $P(z, \alpha)$ in the following form,

$$
C I_{\nu}=P_{n}^{\nu}(z, \alpha) \pm q_{1-\frac{\beta}{2}}\left\{\left(\int_{\mathbb{R}} K_{\nu}^{2}(y) d y\right) P_{n}^{\nu}(z, 2 \alpha)-\left(P_{n}^{\nu}(z, \alpha)\right)^{2}\right\}^{\frac{1}{2}} / \sqrt{n}
$$

as long as $\left(\int_{\mathbb{R}} K_{\nu}^{2}(y) d y\right) P_{n}^{\nu}(z, 2 \alpha)-\left(P_{n}^{\nu}(z, \alpha)\right)^{2}>0$. Denote this inequality by $(\mathbf{C})$. If it is not verified we increase the size of the sample from $n$ to $n+1$. If for all $n$ the inequality (C) is not satisfied, we vary $\nu$ to increase $\int_{\mathbb{R}} K_{\nu}^{2}(y) d y$. There exists then $\nu \in \Gamma$ and a integer $n_{0}$ from which the interval $C I_{\nu}$ is defined. Indeed: let $\nu_{k} \in \Gamma$ be a sequence such that $\int_{\mathbb{R}} K_{\nu_{k}}^{2}(y) d y$ converges to 1 . Then, since $P(z, 2 \alpha)-(P(z, \alpha))^{2}>0$ according to the empirical estimator, we have $\left(\int_{\mathbb{R}} \mathrm{K}_{\nu_{\mathrm{k}}}^{2}(\mathrm{y}) \mathrm{dy}\right) P(z, 2 \alpha)-(P(z, \alpha))^{2}>0$ for $k$ large enough greater than or equal to $k_{0}$. The inequality $(\mathbf{C})$ is then verified from an integer $n_{0}$ and for $\nu=\nu_{k_{0}}$, under the conditions of the Theorems 2.7, 2.8 in Ciss et al. (2015) and the convergence in mean square of $P_{n}^{\nu}(z, \alpha)$ to $P(z, \alpha)$ [Theorems 2.7, 2.8 in Ciss et al., 2015]. We conclude that, the length of the confidence interval $C I_{\nu}$ associated with our estimator is asymptotically lower than that of the empirical estimator, the coefficient being $\left(\int_{\mathbb{R}} K_{\nu}^{2}(y) d y\right)^{1 / 2}<1$.

Remark 2. Consider the inequality $(\mathbf{C})$. The quantity $P_{\nu}=\int_{\mathbb{R}} K_{\nu}^{2}(y) d y$ may be considered as a weight placed in $P(z, 2 \alpha)$. Greater weight is attached to higher poverty line (It is even heavier than the ratio or proportion $Q_{n}=\frac{P_{n}^{\nu}(z, \alpha)}{P_{n}^{\nu}(z, 2 \alpha)}$ is high. So, in order to perform a normality test or to construct a confidence interval of $P(z, \alpha)$, we must calculate $Q_{n}(z, \nu, \alpha)$ for a kernel $K_{\nu_{0}}$. If $P_{\nu_{0}}>Q_{n}(z, \alpha)$, we determine $C I_{\nu_{0}}$ by the equality (3), otherwise a greater weight kernel $K_{\nu}$ is chosen such that the inequality is verified. 
Y. Ciss and A. Diakhaby, Afrika Statistika, Vol. 11(2), 2016, pages 965-980. Asymptotic normality of non-parametric estimator for the FGT poverty index with when the parameter is strictly

Remark 3. Consider the following family of kernels $K_{\nu}(x)=\frac{K(\nu x)}{\int_{\mathbb{R}} K(\nu x)}, \nu>0$. We verify that they satisfy the hypotheses $\mathbf{H}_{\mathbf{1}}-\mathbf{H}_{\mathbf{7}}$. Moreover $\int_{\mathbb{R}} K_{\nu}^{2}(y) d y=\nu \int_{\mathbb{R}} K^{2}(y) d y$. Therefore $\Gamma$ can be the interval $] 0, \frac{1}{\int_{\mathbb{R}} K^{2}(y) d y}[$.

\section{Application}

As an illustration, we will determine the confidence intervals containing $P(z, 0.05)$ for different regions of Senegal and look down results obtained with those of the empirical estimator. The base used is focused on ESAM/1996 (Senegal household Survey) provided by the National Statistics office. Incomes vary from 100 CFA to Millions CFA in the region of DAKAR. The poverty line was $z_{0}=143080$ CFA.

\begin{tabular}{|c||l|l|l|l|l|}
\hline Région & $Q_{n}$ & $P_{\nu}$ & $C I$ & $\widehat{P}\left(z_{0}, 0.05\right)$ & CI(empirical) \\
\hline TAMBA & 0.8113071 & 0.85 & {$[0.764249,0.8122033]$} & 0.7882297 & {$[0.04316811,1.53329]$} \\
TAOURBL & 0.7912961 & 0.80 & {$[0.753301,0.781406]$} & 0.7673563 & {$[-0.00505,1.539766]$} \\
FATICK & 0.6862596 & 0.70 & {$[0.6509295,0.6751269]$} & 0.6629407 & {$[-0.2159264,1.541808]$} \\
THIES & 0.6783842 & 0.70 & {$[0.6429352,0.6739992]$} & 0.6657269 & {$[-0.2167708,1.548225]$} \\
KAOLACK & 0.6740153 & 0.70 & {$[0.63891,0.6639484]$} & 0.6514305 & {$[-0.2365174,1.539378]$} \\
SAINT-LOUIS & 0.6558817 & 0.70 & {$[0.6118941,0.655739]$} & 0.634934 & {$[-0.2650484,1.534916]$} \\
LOUGA & 0.6546964 & 0.70 & {$[0.6131567,0.649072]$} & 0.6310075 & {$[-0.26885502,1.530565]$} \\
ZIGUINCHOR & 0.6464364 & 0.65 & {$[0.614602,0.6283142]$} & 0.6214747 & {$[-0.2793551,1.522304]$} \\
DAKAR & 0.5992101 & 0.60 & {$[0.5758254,0.5814286]$} & 0.5833061 & {$[-0.342700002,1.509312]$} \\
\hline
\end{tabular}

Table 1. Confidence interval containing $P(z, 0.05)$ for different regions

Remark 4. For Ziguinchor, 0.5833061 is not in our confidence interval for the weight setting. If we take a weight equal to 0.65 we have $C I=[0.5561609,0.601093]$ of broader scope but certainly contained in the empirical interval containing the point 0.5833061 . The same applies to DAKAR with a weight equal to 0.40

$C I=[0.3211539,0.3468787]$ contains the point 0.336986 . We have shown with this application that our methodology is not only more efficient than the empirical estimator but also provides confidence intervals smaller scope containing the parameter $P(z, \alpha)$.

\section{Details of the Proofs}

We recall that the power function with $\alpha$, for $\alpha \in[0,1]$ particulary in $\alpha \in] 0,1[$ is the simplest example of Lipschitz function of order $\alpha$ or function $\alpha$-hôldérienne. Indeed, for all reals $x$ and $y$ strictly positive we have

$$
0 \leq(x+y)^{\alpha}-x^{\alpha} \leq y^{\alpha}
$$

Since a factorization of the above expression gives

$$
(x+y)^{\alpha}-x^{\alpha}=y^{\alpha}\left[\left(1+\frac{x}{y}\right)^{\alpha}-\left(\frac{x}{y}\right)^{\alpha}\right]=y^{\alpha}\left[(1+X)^{\alpha}-X^{\alpha}\right] \quad \text { with } \quad X=\frac{x}{y},
$$

it then suffices to study the function $g$ defined in the following form

$$
g(X)=(1+X)^{\alpha}-X^{\alpha} .
$$


Y. Ciss and A. Diakhaby, Afrika Statistika, Vol. 11(2), 2016, pages 965-980. Asymptotic normality of non-parametric estimator for the FGT poverty index with when the parameter is strictly between 0 and 1

The power function with $\alpha>0$ is strictly increasing and differentiable. So, $g(X)>0$. The derivative of $g$ is given by:

$$
g^{\prime}(X)=\alpha(1+X)^{\alpha-1}-\alpha X^{\alpha-1} .
$$

The power function with $\beta<0$ is decreasing and $\alpha-1<0$ therefore

$$
g^{\prime}(X)<0 .
$$

The function $g$ is decreasing and, for all $X$, therefore

$$
g(X) \leq g(0)=1 .
$$

The expression $g(X)$ is between 0 and 1 . This shows the limits of $(x+y)^{\alpha}-x^{\alpha}$.

We say that $f$ is lipschitz of order $\alpha$ on an interval $I$ if there exists a real $M$ such that, for all real number $x_{1}$ and for all real number $y$ in $I$

$$
\left|f\left(x_{1}\right)-f\left(y_{1}\right)\right| \leq M\left|x_{1}-y_{1}\right|^{\alpha} .
$$

Therefore letting $x_{1}=x$ and $y=y_{1}-x_{1}$ and with $f\left(x_{1}\right)=x_{1}^{\alpha}$ we obtain the result.

\section{Proof of Lemma 1}

It is a generalisation of the Lemma 2.9 in Ciss et al. (2015). We assume $\mathbf{C}_{\mathbf{1}}$ holds.

Let $\delta>0$. Define,

$$
\begin{aligned}
I_{n}(x, y) & =h^{-3} \int_{-\infty}^{+\infty}\left|K\left(\frac{u-x+\theta_{1} h}{h}\right) K\left(\frac{u-y+\theta_{2} h}{h}\right) K\left(\frac{u-t+\theta_{3} h}{h}\right)\right| f(u) d u \\
& =\int_{-\infty}^{+\infty}\left(h^{-1} K\left(\frac{v}{h}\right)\right) \mid\left(h^{-1} K\left(\frac{v+x-\theta_{1} h-y+\theta_{2} h}{h}\right)\right) \\
& \times\left(h^{-1} K\left(\frac{v+x-\theta_{1} h-t+\theta_{3} h}{h}\right)\right) \mid f\left(x+v-\theta_{1} h\right) d v \\
& =\int_{|v| \leq \delta}+\int_{|v|>\delta} .
\end{aligned}
$$

Since $f$ is continuous, it is so bounded on $I=[x-\delta, x+\delta]$. We assume $n$ large enough such that $x+v \pm \theta_{1} h \in I$. Then

$$
\begin{aligned}
\int_{|v| \leq \delta} & \leq \sup _{|v| \leq \delta} f\left(x+v-\theta_{1} h\right) \int_{-\frac{\delta}{h} \leq u \leq \frac{\delta}{h}}|K(u)| \mid K\left(\frac{x-\theta_{1} h-y+\theta_{2} h}{h}+u\right) \\
& \times h^{-1} K\left(\frac{x-\theta_{1} h-t+\theta_{3} h}{h}+u\right) h^{-1} \mid d u \\
& =\sup _{|v| \leq \delta} f\left(x+v-\theta_{1} h\right) \int_{-\infty}^{+\infty} \chi_{-\frac{\delta}{h} \leq u \leq \frac{\delta}{h}}|K(u)| \mid K\left(\frac{x-\theta_{1} h-y+\theta_{2} h}{h}+u\right) h^{-1} \\
& \times K\left(\frac{x-\theta_{1} h-t+\theta_{3} h}{h}+u\right) h^{-1} \mid d u .
\end{aligned}
$$

For all $u$

$$
\lim _{n \rightarrow+\infty}\left|K\left(\frac{x-\theta_{1} h-y+\theta_{2} h}{h}+u\right) h^{-1}\right|=0 .
$$


Y. Ciss and A. Diakhaby, Afrika Statistika, Vol. 11(2), 2016, pages 965-980. Asymptotic normality of non-parametric estimator for the FGT poverty index with when the parameter is strictly

Write

$$
\begin{aligned}
& \left|K\left(\frac{x-\theta_{1} h-y+\theta_{2} h}{h}+u\right) h^{-1}\right|= \\
& \left|\left(\frac{x-\theta_{1} h-y+\theta_{2} h}{h}+u\right) K\left(\frac{x-\theta_{1} h-y+\theta_{2} h}{h}+u\right)\right|\left|\frac{1}{x-\theta_{1} h-y+\theta_{2} h+h u}\right| .
\end{aligned}
$$

We have

$$
\left|\frac{1}{x-\theta_{1} h-y+\theta_{2} h+h u}\right|=\frac{1}{|x-y|\left|1-\frac{\theta_{1}-\theta_{2}-u}{x-y} h\right|} .
$$

Since $|u| \leq \frac{\delta}{h}$ we may choose $\delta$ small enough such that for $n \geq n_{0}$ we have

$$
\left|\frac{\theta_{1}-\theta_{2}-u}{x-y} h\right| \leq \frac{3(h)_{n_{0}}+\delta}{|x-y|}=\eta_{1}<1
$$

Therefore

$$
\left|\frac{1}{x-\theta_{1} h-y+\theta_{2} h+h u}\right| \leq \frac{1}{|x-y|\left(1-\eta_{1}\right)},
$$

since $\mathbf{H}_{\mathbf{3}}$ implies there exists a constant $B$ such that

$$
\left|\left(\frac{x-\theta_{1} h-y+\theta_{2} h}{h}+u\right) K\left(\frac{x-\theta_{1} h-y+\theta_{2} h}{h}+u\right)\right| \leq B .
$$

Then we have

$$
\chi_{-\frac{\delta}{h} \leq u \leq \frac{\delta}{h}}(u)\left|K\left(\frac{x-\theta_{1} h-y+\theta_{2} h}{h}+u\right) h^{-1}\right| \leq \frac{B}{|x-y|\left(1-\eta_{1}\right)} .
$$

Similarly, there exists a constant $C$ such that

$$
\left|\left(\frac{x-\theta_{1} h-t+\theta_{3} h}{h}+u\right) K\left(\frac{x-\theta_{1} h-t+\theta_{3} h}{h}+u\right)\right| \leq C
$$

and

$$
\chi_{-\frac{\delta}{h} \leq u \leq \frac{\delta}{h}}(u)\left|K\left(\frac{x-\theta_{1} h-t+\theta_{3} h}{h}+u\right) h^{-1}\right| \leq \frac{C}{|x-t|(1-\eta 2)} .
$$

Therefore, if $\delta$ is small enough and $n$ sufficiently large we have, for $-\frac{\delta}{h} \leq u \leq \frac{\delta}{h}$ $|K(u)|$ being integrable, by dominated convergence theorem, the integral in the right-hand side of (4) tends to zero as $n \rightarrow+\infty$, uniformly with respect to $\left(\theta_{1}, \theta_{2}, \theta_{3}\right)$. Hence

$$
\int_{|v| \leq \delta} \rightarrow 0 \text { as } n \rightarrow+\infty \quad \text { uniformly with respect to }\left(\theta_{1}, \theta_{2}, \theta_{3}\right) \text {. }
$$

Let $\int_{|v|>\delta}$; write in the form

$$
\begin{aligned}
\int_{|v|>\delta} & =\int_{\left|v-\theta_{1} h\right|>\delta} \mid v h^{-1} K\left(\frac{v}{h}\right)\left(h^{-1} K\left(\frac{v+x-\theta_{1} h-y+\theta_{2} h}{h}\right)\right. \\
& \times\left(h^{-1} K\left(\frac{v+x-\theta_{1} h-t+\theta_{3} h}{h}\right)\right) \frac{f\left(x+v-\theta_{1} h\right)}{v} \mid d v .
\end{aligned}
$$


Y. Ciss and A. Diakhaby, Afrika Statistika, Vol. 11(2), 2016, pages 965-980. Asymptotic normality of non-parametric estimator for the FGT poverty index with when the parameter is strictly between 0 and 1

We obtain

$$
\begin{aligned}
\int_{|v|>\delta} & \leq \frac{2}{\delta} \sup _{|v|>\delta}\left|\frac{v}{h} K\left(\frac{v}{h}\right)\right| \int_{|v|>\delta}\left(h^{-1} K\left(\frac{v+x-\theta_{1} h-y+\theta_{2} h}{h}\right)\right) \\
& \times\left(h^{-1} K\left(\frac{v+x-\theta_{1} h-t+\theta_{3} h}{h}\right)\right)\left|f\left(x+v-\theta_{1} h\right)\right| d v .
\end{aligned}
$$

Making the change of variable

$$
v+x-\theta_{1} h=u
$$

Then

$$
\begin{aligned}
\int_{|v|>\delta} & \leq \frac{2}{\delta-\theta_{1} h} \sup _{|v|>\delta}\left|\frac{v}{h} K\left(\frac{v}{h}\right)\right| \int_{\mathbb{R}}\left|h^{-1} K\left(\frac{u-y+\theta_{2} h}{h}\right)\left(h^{-1} K\left(\frac{u-t+\theta_{3} h}{h}\right)\right)\right| f(u) d u \\
& \leq \int_{\mathbb{R}}\left|h^{-1} K\left(\frac{u-y+\theta_{2} h}{h}\right)\left(h^{-1} K\left(\frac{u-t+\theta_{3} h}{h}\right)\right)\right| f(u) d u
\end{aligned}
$$

and this quantity in right-side tends to zero as $n \rightarrow+\infty$ uniformly with respect to $\left(\theta_{2}, \theta_{3}\right)$ according to Lemma 2.9 in Ciss et al. (2015) (when we replace $K$ by $|K|$ ) and $\left(\mathbf{H}_{\mathbf{3}}\right)$ (valid under assumption $\mathbf{C}_{\mathbf{1}}$ or $\mathbf{C}_{\mathbf{2}}$ ). Since

$$
\sup _{|v|>\delta}\left|\frac{v}{h} K\left(\frac{v}{h}\right)\right| \rightarrow 0 \quad \text { as } \quad n \rightarrow+\infty,
$$

we have

$$
\left|\int_{|v|>\delta}\right| \rightarrow 0 \quad \text { as } \quad n \rightarrow+\infty,
$$

the proof of the Lemma 1 is complete.

\section{Proof of Lemma 2}

We Suppose condition $\mathbf{C}_{\mathbf{1}}$ is verified. Let $\Delta=[0, b] \times[0, b] \times[0, b]$. We can write for all $z \in[0, b]$

$$
\begin{aligned}
\sum_{0 \leq i \neq j \neq l \neq i \leq\left[\frac{z}{h}\right]}\left(1-\frac{i h}{z}\right)^{\alpha}\left(1-\frac{j h}{z}\right)^{\alpha}\left(1-\frac{l h}{z}\right)^{\alpha} & \\
\times \int_{\mathbb{R}} \mid & K\left(\frac{u-i h}{h}\right) K\left(\frac{u-j h}{h}\right) K\left(\frac{u-l h}{h}\right) \mid f(u) d u \\
& \leq M_{1}^{3} h^{3 \alpha} \iiint_{\{(x, y, t) \in \Delta:|x-y||x-t||t-y|>0\}} \Phi_{n}(x, y, t) d x d y d t
\end{aligned}
$$

where

$$
\begin{aligned}
\Phi_{n}(x, y, t) & =\frac{1}{h^{3}} \sum_{0 \leq i \neq j \neq l \neq i \leq\left[\frac{z}{h}\right]} \chi_{\Delta_{h, i}}(x) \chi_{\Delta_{h, j}}(y) \chi_{\Delta_{h, l}}(t) \\
& \times \int_{\mathbb{R}}\left|K\left(\frac{u-i h}{h}\right) K\left(\frac{u-j h}{h}\right) K\left(\frac{u-l h}{h}\right)\right| f(u) d u .
\end{aligned}
$$


Y. Ciss and A. Diakhaby, Afrika Statistika, Vol. 11(2), 2016, pages 965-980. Asymptotic normality of non-parametric estimator for the FGT poverty index with when the parameter is strictly between 0 and 1

Let $(x, y, t) \in \Delta_{h, i} \times \Delta_{h, j} \times \Delta_{h, l} \quad i \neq j \neq l \neq i$ with the representations:

$$
x=h i+\theta_{1} h, \quad y=h j+\theta_{2} h \quad t=h l+\theta_{3} h \quad 0 \leq \theta_{i}<1, \quad i=1,2,3
$$

(7) becomes

$$
\frac{1}{h^{3}} \int_{\mathbb{R}}\left|K\left(\frac{u-i h}{h}\right) K\left(\frac{u-j h}{h}\right) K\left(\frac{u-l h}{h}\right)\right| f(u) d u .
$$

Let $\delta=\min \left(\frac{|x-y|}{2}, \frac{|x-t|}{2}, \frac{|t-y|}{2}\right)$. With the change of variable $v=u-x-\theta_{1} h$ we split the integral above in the following form:

$$
\frac{1}{h^{3}} \int_{\mathbb{R}}\left|K\left(\frac{v}{h}\right) K\left(\frac{x-\theta_{1} h-y+\theta_{2} h}{h}\right) K\left(\frac{x-\theta_{1} h-t+\theta_{3} h}{h}\right)\right| f(u) d u=\int_{|v| \leq \delta}+\int_{|v|>\delta} .
$$

Then, we have

$$
\begin{aligned}
& \iiint_{\{(x, y, t) \in \Delta:|x-y||x-t||t-y|>0\}} \Phi_{n}(x, y, t) d x d y d t \\
& \leq \iiint_{\{(x, y, t) \in \Delta:|x-y||x-t||t-y|>0\}} \sum_{0 \leq i \neq j \neq l \neq i \leq\left[\frac{z}{h}\right]} \chi_{\Delta_{h, i} \times \Delta_{h, j} \times \Delta_{h, l}}(x, y, t)\left(\int_{|v| \leq \delta}+\int_{|v|>\delta}\right) .
\end{aligned}
$$

The proof is conducted as follow:

First consider

$$
\iiint_{\{(x, y, t 1) \in \Delta:|x-y||x-t||t-y|>0\}} \sum_{0 \leq i \neq j \neq l \neq i \leq\left[\frac{z}{h}\right]} \chi_{\Delta_{h, i} \times \Delta_{h, j} \times \Delta_{h, l}}(x, y, t)\left(\int_{|v| \leq \delta}\right) .
$$

Let $A=\sup _{x \in \mathbb{R}} f(x)$. The notations being as in the proof of Lemma 2.9 in Ciss et al. (2015) and we suppose $h \leq \frac{b}{4}$ for $n$ large enough with $\delta=\frac{z}{2}$. We have, in accordance with inequality $(4)$

$$
\begin{aligned}
\int_{|v| \leq \delta} & \leq A \int_{-\infty}^{+\infty} \chi_{-\frac{\delta}{h} \leq u \leq \frac{\delta}{h}}|K(u)|\left|K\left(\frac{x-\theta_{1} h-y+\theta_{2} h}{h}+u\right) h^{-1}\right| \\
& \times\left|K\left(\frac{x-\theta_{1} h-t+\theta_{3} h}{h}+u\right) h^{-1}\right| d u .
\end{aligned}
$$

For all $u$

$$
\lim _{n \rightarrow+\infty}\left|K\left(\frac{x-\theta_{1} h-y+\theta_{2} h}{h}+u\right) h^{-1}\right|=0 .
$$

Write

$$
\begin{aligned}
\left|K\left(\frac{x-\theta_{1} h-y+\theta_{2} h}{h}+u\right) h^{-1}\right| & =\mid K\left(\frac{x-\theta_{1} h-y+\theta_{2} h}{h}+u\right) \\
& -K\left(\frac{2 b+x-\theta_{1} h-y+\theta_{2} h}{h}+u\right) \\
& +K\left(\frac{2 b+x-\theta_{1} h-y+\theta_{2} h}{h}+u\right) \mid h^{-1} \\
& \leq\left(\lambda\left(\frac{2 b}{h}\right)+\left|K\left(\frac{2 b+x-\theta_{1} h-y+\theta_{2} h}{h}+u\right)\right|\right) h^{-1} .
\end{aligned}
$$


Y. Ciss and A. Diakhaby, Afrika Statistika, Vol. 11(2), 2016, pages 965-980. Asymptotic normality of non-parametric estimator for the FGT poverty index with when the parameter is strictly between 0 and 1

Express

$$
\begin{aligned}
& \left|K\left(\frac{2 b+x-\theta_{1} h-y+\theta_{2} h}{h}+u\right)\right| h^{-1} \\
& =\left|\frac{2 b+x-y+h u}{h}\right|\left|K\left(\frac{2 b+x-\theta_{1} h-y+\theta_{2} h}{h}+u\right)\right| \frac{1}{|2 z+x-y+h u|} .
\end{aligned}
$$

Let $B=\sup _{y \in \mathbb{R}}|y||K(y)|$ and $C=\sup _{y \in \mathbb{R}}|K(y)|$, then, we have

$$
\left|\frac{2 b+x-y+h u}{h}\right|\left|K\left(\frac{2 z+x-\theta_{1} h-y+\theta_{2} h}{h}+u\right)\right| \leq B+2 C .
$$

Therefore, we have

$$
\left|K\left(\frac{2 b+x-\theta_{1} h-y+\theta_{2} h}{h}+u\right)\right| h^{-1} \leq \frac{B+2 C}{|2 b+x-y+h u|} .
$$

Hence

$$
\left|K\left(\frac{x-\theta_{1} h-y+\theta_{2} h}{h}+u\right) h^{-1}\right| \leq \lambda\left(\frac{2 b}{h}\right)+\frac{B+2 C}{|2 b+x-y+h u|} .
$$

Similarly, we have

$$
\left|K\left(\frac{x-\theta_{1} h-t+\theta_{3} h}{h}+u\right) h^{-1}\right| \leq \lambda\left(\frac{2 b}{h}\right)+\frac{B+2 C}{|2 b+x-t+h u|} .
$$

We conclude that for $h$ small enough

$$
\int_{|v| \leq \delta} \leq \frac{A}{|2 b+x-y+h u|} \int_{\mathbb{R}}|K(u)|(B+2 C) d u<\frac{A D}{|2 b+x-y+h u|}
$$

$D$ being the finite bound of $\int_{\mathbb{R}}|K(u)|(B+2 C) d u$.

We have

$$
\int_{|v| \leq \delta} \leq \frac{A D}{|2 b+x-y+h u|}+O(h) .
$$

Since $-\delta \leq h u \leq \delta$ we have

$$
\frac{b}{4} \leq|2 b+x-y+h u|, \text { et } \frac{b}{4} \leq|2 b+x-t+h u| .
$$

Hence

$$
\int_{|v| \leq \delta} \leq \frac{16 A D}{b^{2}}+O(h)
$$

This inequality is true for all

$$
(x, y, t) \in\{(x, y, t) \in \Delta:|x-y \| x-t||t-y|>0\} .
$$


Y. Ciss and A. Diakhaby, Afrika Statistika, Vol. 11(2), 2016, pages 965-980. Asymptotic normality of non-parametric estimator for the FGT poverty index with when the parameter is strictly

For all $u$ we have according to the proof of the Lemma 2.3 in Ciss et al. (2015)

$$
\begin{aligned}
\sup _{\left(\theta_{1}, \theta_{2}, \theta_{3}\right) \in[0,1]^{3}} & \int_{-\infty}^{+\infty} \chi_{-\frac{\delta}{h} \leq u \leq \frac{\delta}{h}}(u)|K(u)|\left|K\left(\frac{x-\theta_{1} h-y+\theta_{2} h}{h}+u\right) h^{-1}\right| \\
& \times\left|K\left(\frac{x-\theta_{1} h-t+\theta_{3} h}{h}+u\right) h^{-1}\right| d u
\end{aligned}
$$

tends to zero as $n \rightarrow+\infty$ except the complement in $\Delta$ of

$$
\{(x, y, t) \in \Delta, x \neq y \neq t \neq x\}
$$

which is $d x d y d t$-measure nul.

Therefore by Lebesgue-dominated convergence theorem

$$
\begin{gathered}
\lim _{n \rightarrow+\infty} \iiint_{\Delta} \sum_{0 \leq i \neq j \neq l \neq i \leq\left[\frac{z}{h}\right]} \chi_{\Delta_{h, i} \times \Delta_{h, j} \times \Delta_{h, l}}(x, y, t)\left(\int_{|v| \leq \delta}\right) d x d y d t \\
=\iiint_{\Delta} \lim _{n \rightarrow+\infty}\left(\int_{|v| \leq \delta}\right) d x d y d t=0 .
\end{gathered}
$$

Consider finally

$$
\iiint_{\{(x, y, t) \in \Delta:|x-y||x-t||t-y|>0\}} \sum_{0 \leq i \neq j \neq l \neq i \leq\left[\frac{z}{h}\right]} \chi_{\Delta_{h, i} \times \Delta_{h, j} \times \Delta_{h, l}}(x, y, t) \int_{|v|>\delta} .
$$

We use expression (3) in the second part of Lemma 2.9 in Ciss et al. (2015) by analogous reasoning, we obtain

$$
\begin{aligned}
\int_{|v|>\delta} & \leq \frac{1}{\delta} \sup _{|v|>\delta}\left|\frac{v}{h}\right|\left|K\left(\frac{v}{h}\right)\right| \int_{|v|>\delta} \mid h^{-1} K\left(\frac{v+x-\theta_{1} h-y+\theta_{2} h}{h}\right) \\
& \times h^{-1} K\left(\frac{v+x-\theta_{1} h-t+\theta_{3} h}{h}\right) \mid f\left(x+v-\theta_{1} h\right) d v .
\end{aligned}
$$

Making a change of variable $u=x+v-\theta_{1} h$, the integral of the right-hand side of (12) does not exceed

$$
\int_{\mathbb{R}} \mid h^{-1} K\left(\frac{u-y+\theta_{2} h}{h}\right) h^{-1} K\left(\frac{u-t+\theta_{3} h}{h} \mid f(u) d u .\right.
$$

Let $\delta \geq h^{\varepsilon}, 0<\varepsilon<\frac{1}{2}$. We have

$$
\begin{aligned}
& \int_{|v|>\delta} \leq \sup _{|v|>h^{\varepsilon}} \frac{|v|}{h^{1+\varepsilon}}\left|K\left(\frac{v}{h}\right)\right| \sup _{\left(\theta_{2}, \theta_{3}\right) \in[0,1]^{2}} \int_{\mathbb{R}} \mid h^{-1} K\left(\frac{u-y+\theta_{2} h}{h}\right) h^{-1} \\
& \times K\left(\frac{u-t+\theta_{3} h}{h} \mid f(u) d u .\right.
\end{aligned}
$$

When writing

$$
\int_{\mathbb{R}}\left|h^{-1} K\left(\frac{u-y+\theta_{2} h}{h}\right) h^{-1} K\left(\frac{u-t+\theta_{3} h}{h}\right)\right| f(u) d u \leq \int_{|v| \leq \bar{\delta}}+\int_{|v|>\bar{\delta}},
$$


Y. Ciss and A. Diakhaby, Afrika Statistika, Vol. 11(2), 2016, pages 965-980. Asymptotic normality of non-parametric estimator for the FGT poverty index with when the parameter is strictly between 0 and 1

with the change of variable $v=u-t+\theta_{3} h$ and $\bar{\delta}=\frac{|t-y|}{2}$ we have according to the proof of Theorem 2.10 in Ciss et al. (2015), under hypothesis $\mathbf{C}_{\mathbf{1}}$ or $\mathbf{C}_{\mathbf{2}}$, for $\bar{\delta}>h^{\varepsilon}$

$$
\mid \int_{|v| \leq \bar{\delta}} \leq \frac{4 A D}{b}+O(h), \quad y \neq t
$$

and

$$
\left|\int_{|v|>\bar{\delta}}\right| \leq \sup _{|v|>h^{\varepsilon}} \frac{|v|}{h^{1+\varepsilon}}\left|K\left(\frac{v}{h}\right)\right| \int_{\mathbb{R}}\left|h^{-1} K\left(\frac{v}{h}\right)\right| f\left(u+y-\theta_{2} h\right) d u .
$$

Since

$$
\int_{\mathbb{R}}\left|h^{-1} K\left(\frac{v}{h}\right)\right| f\left(u+y-\theta_{2} h\right) d u
$$

is bounded by $A \int_{\mathbb{R}}|K(u)| d u$, we deduce that the right-hand side of(12) is bounded except on set, $\bar{\delta}=0$. Hence

$$
\int_{|v|>\delta}
$$

is bounded except for $\{(x, y, t) \in \Delta: \delta=0\}$ which is ( $d x d y d t)$-measure nul. $\Delta$ being bounded, hypothesis $\mathbf{H}_{\mathbf{7}}$ implies that the integral (10) tends to zero as $n \rightarrow+\infty$. Consequently

$$
\lim _{n \rightarrow+\infty} \iint_{\Delta} \int_{\mathbb{R}} \rightarrow 0, \quad n \rightarrow+\infty
$$

since $\Delta$ is bounded. The proof of lemma is then complete.

\section{Proof of Theorem 1}

For sake of simplicity in the notations, $K$ stands for $K_{\nu}$ and $P_{n}$ for $P_{n}^{\nu}$. It is sufficient to prove: on the one hand,

$$
\text { 1). } \sqrt{n}\left(\mathbb{E}\left(P_{n}(z, \alpha)-P(z, \alpha)\right) \rightarrow n \quad \text { as } \quad n \rightarrow+\infty\right.
$$

and on the other hand,

$$
\text { 2). } \frac{P_{n}(z, \alpha)-\mathbb{E}\left(P_{n}(z, \alpha)\right)}{\sqrt{\mathbb{V}\left(P_{n}(z, \alpha)\right)}} \rightarrow N(0,1) \quad \text { as } \quad n \rightarrow+\infty .
$$

Let $x_{0}$ be the infimum of the support of $f$. Let's firstly, observe that $F(z)$ is bounded. Let $\bar{\Delta}_{h, i}=\Delta_{h, i} \cap[0, z]$; and $\chi_{B}$ the indicator function of $B$. Let $z \in[0, b]$. We have

$$
\mathbb{E}\left(P_{n}(z, \alpha)\right)=\sum_{i=1}^{\left[\frac{z}{h}\right]}\left(1-\frac{i h}{z}\right)^{\alpha} \int_{\mathbb{R}} K(u) f(u h-i h) d u
$$

which can be written in the following form

$$
\begin{aligned}
& \int_{0}^{z} \sum_{i=1}^{\left[\frac{z}{h}\right]} \chi_{\bar{\Delta}_{h, i}}(x)\left(1-\frac{i h}{z}\right)^{\alpha} \int_{-\infty}^{+\infty} K(u) f(u h-i h) d u d x \\
& +\left(h\left(\left[\frac{z}{h}\right]+1\right)-z\right)\left(1-\frac{h\left[\frac{z}{h}\right]}{z}\right)^{\alpha} \int_{-\infty}^{+\infty} K(u) f\left(u h+\frac{h\left[\frac{z}{h}\right]}{z}\right) d u .
\end{aligned}
$$


Y. Ciss and A. Diakhaby, Afrika Statistika, Vol. 11(2), 2016, pages 965-980. Asymptotic normality of non-parametric estimator for the FGT poverty index with when the parameter is strictly between 0 and 1

We have

$$
\begin{gathered}
\sup _{z \in \mathbb{R}} \mid\left(h\left(\left[\frac{z}{h}\right]+1\right)-z\right) \\
\left(1-\frac{h\left[\frac{z}{h}\right]}{z}\right)^{\alpha} \int_{-\infty}^{+\infty} K(u) f\left(u h+\frac{h\left[\frac{z}{h}\right]}{z}\right) d u \mid \\
\leq 2 M_{1} h^{2+\alpha} \sup _{x \in \mathbb{R}} f(x) \int_{-\infty}^{+\infty}|K(u)| d u .
\end{gathered}
$$

Since we have $\left|h\left(\left[\frac{z}{h}\right]+1\right)-z\right| \leq 2 h$. Because of assumption $\mathbf{H}_{2}$, we can write

$$
P(z, \alpha)=\int_{0}^{z}\left(1-\frac{x}{z}\right)^{\alpha} K(u) d u f(x) d x .
$$

Let $x \in \bar{\Delta}_{h, i}$. By considering the terms (13) and (15), we get

$$
\begin{aligned}
& \left|\left(1-\frac{i h}{z}\right)^{\alpha} \int_{-\infty}^{+\infty} K(u) f(u h-i h) d u-\left(1-\frac{x}{z}\right)^{\alpha} K(u) d u f(x)\right| \\
& =\left|\int_{-\infty}^{+\infty}\left[\left(1-\frac{i h}{z}\right)^{\alpha} f(u h-i h)-\left(1-\frac{x}{z}\right)^{\alpha} f(x)\right] K(u) d u\right| \\
& \leq \int_{-\infty}^{+\infty}\left|\left(1-\frac{i h}{z}\right)^{\alpha}-\left(1-\frac{x}{z}\right)^{\alpha}\right| f(x)|K(u)| d u \\
& +\int_{-\infty}^{+\infty}\left|\left(1-\frac{i h}{z}\right)^{\alpha}\right||f(u h-i h)-f(x)||K(u)| d u .
\end{aligned}
$$

Let $x \in \Delta_{h, i}$, we have, by the lipschitz condition applied to the function,

$$
\begin{gathered}
g(x)=\left(1-\frac{x}{z}\right)^{\alpha} \\
\left|\left(1-\frac{i h}{z}\right)^{\alpha}-\left(1-\frac{x}{z}\right)^{\alpha}\right| \leq M_{1}|i h-x|^{\alpha} \leq M_{1} h^{\alpha} .
\end{gathered}
$$

Therefore, denoting by $I_{1}^{i}(x)$ the first integral of the right hand-side of (16) and

$$
I_{1}(x)=\sum_{i=1}^{\left[\frac{z}{h}\right]} \chi_{\bar{\Delta}_{h, i}}(x) I_{1}^{i}(x)
$$

We have

$$
\int_{0}^{z} I_{1}(x) d x \leq M_{1} h^{\alpha} \int_{0}^{z}\left(\int_{-\infty}^{+\infty} f(x)|K(u)| d u\right) d x=M_{1} h^{\alpha}\left(\int_{-\infty}^{+\infty}|K(u)| d u\right) F(z) .
$$

Denoting by $I_{2}^{i}(x)$ the second integral of the right hand-side of (16) and

$$
I_{2}(x)=\sum_{i=1}^{\left[\frac{z}{h}\right]} \chi_{\bar{\Delta}_{h, i}}(x) I_{2}^{i}(x)
$$

Applying the lipschitz condition to the points $i h$ and $z$. We have

$$
I_{2}^{i}(x) \leq M_{1} h^{\alpha}\left(\int_{-\infty}^{+\infty}|f(u h-i h)-f(i h)||K(u)| d u+\int_{-\infty}^{+\infty}|f(i h)-f(x)||K(u)| d u\right)
$$


Y. Ciss and A. Diakhaby, Afrika Statistika, Vol. 11(2), 2016, pages 965-980. Asymptotic normality of non-parametric estimator for the FGT poverty index with when the parameter is strictly

$$
\begin{aligned}
\int_{0}^{z} I_{2}(x) d x & \leq M_{1} h^{\alpha}\left(\sum_{i=1}^{\left[\frac{z}{h}\right]} h \int_{-\infty}^{+\infty}\left|f\left(u h+x-\theta_{1} h\right)-f(x)\right|\right. \\
& \left.\times|K(u)| d u+\varepsilon \int_{-\infty}^{+\infty}|K(u)| d u d x\right) \\
& \leq C M_{1} h^{\gamma+1} z \int_{0}^{z}\left(\int_{\mathbb{R}}\left(|u|^{\gamma}+1\right)|K(u)| d u d x .\right.
\end{aligned}
$$

Since

$$
\int_{0}^{z} I_{1}(x) d x \leq M_{1} h^{\alpha} \int_{0}^{z}\left(\int_{-\infty}^{+\infty} f(x)|K(u)| d u\right) d x=M_{1} h^{\alpha}\left(\int_{-\infty}^{+\infty}|K(u)| d u\right) F(z)
$$

we get, together with the right hand-side of (14)

$$
\begin{aligned}
\sqrt{n}\left(\mathbb{E}\left(P_{n}(z, \alpha)-P(z, \alpha)\right)\right. & \leq h^{\gamma} \sqrt{n}\left\{C M_{1} h^{\alpha} z \int_{0}^{z}\left(\int_{\mathbb{R}}\left(|u|^{\gamma}+1\right)|K(u)| d u\right) d x\right. \\
& +2 M_{1} h^{\alpha}(2-\gamma) \sup _{x \in \mathbb{R}} f(x) \int_{-\infty}^{+\infty}|K(u)| d u \\
& \left.+2 M_{1} h^{\alpha}{ }^{(1-\gamma)} \alpha\left(\int_{-\infty}^{+\infty}|K(u)| d u\right) F(z)\right\} .
\end{aligned}
$$

The integrals in the braces exist. Hence the first part is proved.

For the second part, we define

$$
\begin{gathered}
U_{i}=\frac{1}{n} \sum_{0 \leq l \leq\left[\frac{z}{h}\right]}\left(1-\frac{l h}{z}\right)^{\alpha} K\left(\frac{X_{i}-l h}{h}\right), \\
i=1 \ldots n, \quad \mu_{i}=\mathbb{E}\left(U_{i}\right) \text { and } \beta_{i}=\mathbb{E}\left(\left|U_{i}-\mu_{i}\right|^{3}\right) .
\end{gathered}
$$

Let $B_{n}=\left(\sum_{i=1}^{n} \beta_{i}\right)^{\frac{1}{3}}$. We shall obtain the statement 2$)$ if, by Liapounov's theorem, we prove

$$
\frac{B_{n}}{\sqrt{\operatorname{Var}\left(P_{n}(z, \alpha)\right)}} \rightarrow 0 \quad \text { as } n \rightarrow+\infty
$$


Y. Ciss and A. Diakhaby, Afrika Statistika, Vol. 11(2), 2016, pages 965-980. Asymptotic normality of non-parametric estimator for the FGT poverty index with when the parameter is strictly between 0 and 1

Consider $n^{3} \mathbb{E}\left(U_{i}^{3}\right)$. We have

$$
\begin{aligned}
n^{3} \mathbb{E}\left(U_{j}^{3}\right) & =\mathbb{E}\left[\left(\sum_{0 \leq l \leq\left[\frac{z}{h}\right]}\left(1-\frac{l h}{z}\right)^{\alpha} K\left(\frac{X_{j}-l h}{h}\right)\right)^{3}\right] \\
& =\mathbb{E}\left[\left\{\sum_{0 \leq l \leq\left[\frac{z}{h}\right]}\left(1-\frac{l h}{z}\right)^{3 \alpha} K^{3}\left(\frac{X_{j}-l h}{h}\right)\right.\right. \\
& +\sum_{0 \leq l \neq i \leq\left[\frac{z}{h}\right]}\left(1-\frac{l h}{z}\right)^{2 \alpha} K^{2}\left(\frac{X_{j}-l h}{h}\right)\left(1-\frac{i h}{z}\right)^{\alpha} K\left(\frac{X_{j}-j h}{h}\right) \\
& +\sum_{0 \leq l \neq i \neq j \neq l \leq\left[\frac{z}{h}\right]}\left(1-\frac{l h}{z}\right)^{\alpha} K\left(\frac{X_{j}-l h}{h}\right)\left(1-\frac{i h}{z}\right)^{\alpha} \\
& \left.\left.\times K\left(\frac{X_{j}-i h}{h}\right)\left(1-\frac{j h}{z}\right)^{\alpha} K\left(\frac{X j-j h}{h}\right)\right\}\right] \\
& \leq \sum_{0 \leq i \leq\left[\frac{z}{h}\right]} \int_{\mathbb{R}}\left(M_{1}^{3} h^{3 \alpha}\left|K^{3}\left(\frac{u-i h}{h}\right)\right| f(u) d u\right. \\
& +\sum_{0 \leq l \neq i \leq\left[\frac{z}{h}\right]} \int_{\mathbb{R}} M_{1}^{3} h^{3 \alpha} K^{2}\left(\frac{u-l h}{h}\right)\left|K\left(\frac{u-j h}{h}\right)\right| f(u) d u \\
& +\sum_{0 \leq l \neq i \neq j \neq l \leq\left[\frac{z}{h}\right]} \int_{\mathbb{R}} M_{1}^{3} h^{3 \alpha}\left|K\left(\frac{u-l h}{h}\right) K\left(\frac{u-i h}{h}\right) K\left(\frac{u-j h}{h}\right)\right| f(u) d u .
\end{aligned}
$$

The first term of the right hand-side of this inequality tends to $\left(\int_{\mathbb{R}}\left|K^{3}(y)\right| d y\right) P(z, 2 \alpha)$ as $n \rightarrow+\infty$. The second term of the right hand-side of this inequality tends to zero as $n \rightarrow+\infty$ because of theorem 2.10 Ciss et al. (2015) in the unidimensional case.

The latter two terms of the right hand-side of this inequality tend to zero as $n \rightarrow+\infty$ because of the Fubini's theorem, the Theorem 2.10 and the Corollary 2.5 Ciss et al. (2015) in the unidimensional case.

Therefore, their limits exist. Hence the Liapounov's condition is satisfied. We have

$$
n^{3} \mathbb{E}\left(\left|U_{i}-\mu_{i}\right|\right)^{3}<+\infty \quad \text { and } \quad B_{n}=\frac{\left(\sum_{i=1}^{n} n^{3} \mathbb{E}\left(\left|U_{i}-\mu_{i}\right|\right)\right)^{1 / 3}}{n} .
$$

Let $c s t$ be a constant such that $\left.n^{3} \mathbb{E}\left(\left|U_{i}-\mu_{i}\right|\right)\right)^{3} \leq$ cst therefore

$$
\begin{aligned}
\frac{B_{n}}{\sqrt{\operatorname{Var}\left(P_{n}(z, \alpha)\right)}} & =\frac{\left(\sum_{i=1}^{n} n^{3} \mathbb{E}\left(\left|U_{i}-\mu_{i}\right|\right)\right)^{1 / 3}}{n \sqrt{\operatorname{Var}\left(P_{n}(z, \alpha)\right)}} \\
& =\frac{n^{1 / 3} c s t}{n \sqrt{\operatorname{Var}\left(P_{n}(z, \alpha)\right)}} .
\end{aligned}
$$

On the other hand, we have

$$
\mathbb{V a r}\left(P_{n}(z, \alpha)\right)=O\left(\frac{1}{n}\right)>0
$$


Y. Ciss and A. Diakhaby, Afrika Statistika, Vol. 11(2), 2016, pages 965-980. Asymptotic normality of non-parametric estimator for the FGT poverty index with when the parameter is strictly between 0 and 1

therefore

$$
\frac{B_{n}}{\sqrt{\operatorname{Var}\left(P_{n}(z, \alpha)\right)}}=O\left(\frac{n^{1 / 3}}{n O\left(\frac{1}{n}\right)}\right) \cong\left(\frac{n^{1 / 3}}{n^{\frac{1}{2}}}\right)
$$

which tends to zero as $n \rightarrow+\infty$. This completes the proof.

\section{Proof of Theorem 2}

We have

$$
\sqrt{n}\left(\mathbb{E}\left(P_{n}^{\nu}(z, \alpha)\right)-P(z, \alpha)\right) \rightarrow 0 \quad \text { as } n \rightarrow+\infty,
$$

by the lemma 2.4 Ciss et al. (2015) unidimensional case. The second part remains unchanged. The proof is complete.

\section{References}

Ciss Y., Dia G. and Diakhaby A. 2015. Non-parametric estimation of income distribution and poverty index in the unidimensional context with $\alpha \in] 0,1[.353: 10,947-952$.

Dia, G., 2009. Asymptotic normality of the kernel poverty measure estimate. 3(1):21-39.

Dia, G., 2008. Estimation nonparamétrique de la distribution des revenus et de l'indice de pauvreté. C. R. Acad. Sci. Paris, Ser. I 346, 907-912.

Foster, J. E. and, Greer, J. and, Thorbecke, E., 1984. A class of decomposable poverty measures. Econometrica. 52, 761-776.

Foster, J. E., Greer, J., Thorbecke, E., 2010. The foster-greer-thorbecke (fgt) poverty measures: Twenty five years later. 8(4), 491-524.

Lo, G.S., 2003. Estimation des lois du revenu et des dépenses dans une base de données de pauvreté. Publications de l'UFR-SAT, LERSTAD N8 UGB Sénégal.

Lo, G.S., Sall, S.T., and Seck, C.T., 2009. Une théorie asymptotique générale des mesures de pauvreté. 32(2), 45-52.

Parzen, E., 1962. On estimation of a probability density function and mode. 33(3), 10651076.

Seck, C.T., 2011. Estimation Non-paramétrique et Convergence Faible des Mesures de Pauvreté. PhD thesis, Université Pierre et Marie Curie.

Seck, C.T. and, Lo, G.S., 2009. Uniform convergence of the non-weighted poverty measures. 38(20), 3697-3704.

Seidl, C., 1988. Poverty measurement: a survey, in: D. bos, c. seidl (eds.), welfare and efficienty in public economics. 71-147. 